\title{
Onset of oscillations in Rayleigh-Benard convection: Horizontally unbounded slab
}

\author{
Boyd F. Edwards and Alexander L. Fetter \\ Institute of Theoretical Physics, Department of Physics, Stanford University, Stanford, California 94305
}

(Received 19 April 1984; accepted 23 July 1984)

\begin{abstract}
Rayleigh-Bénard convection in a laterally unbounded classical fluid layer with low Prandtl number $\mathscr{P}$ (ratio of kinematic viscosity to thermal diffusivity) is reexamined. An amplitude expansion with only a few normal modes yields lateral oscillations of the convective rolls, which are therefore only weakly nonlinear. For free boundary conditions, additional modes (absent for rigid boundaries) lead to long wavelength ("hydrodynamic") oscillations, with explicit nonlinear distortions in the velocity and temperature fields. For oscillations with rigid boundaries, the finite critical wavenumbers are approximately independent of $\mathscr{P}$ for small $\mathscr{P}$, and the calculated Rayleigh number, frequency, and wavenumber at onset agree well with observations in air. Discrepancies with experiments in dilute superfiuid ${ }^{3} \mathrm{He}-{ }^{-4} \mathrm{He}$ systems with small aspect ratios (ratio of horizontal to vertical dimensions) suggest that lateral boundaries or two-fluid effects play an important role in these systems.
\end{abstract}

\section{INTRODUCTION}

Recent studies ${ }^{1-3}$ of the onset of convection in dilute superfluid ${ }^{3} \mathrm{He}-{ }^{4} \mathrm{He}$ mixtures show similarity to one-component classical fluids. At low temperatures, the essentially constant ${ }^{4} \mathrm{He}$ chemical potential effectively eliminates the ${ }^{3} \mathrm{He}$ concentration as an independent degree of freedom; in practice, deviations from the classical one-component fluid equations are small. Further experiments ${ }^{4.5}$ in the nonlinear regime have emphasized the onset of oscillations. The present work seeks a corresponding theoretical description. As the two-fluid effects are small at onset of convection, we continue to use the classical single-fluid equations in the Oberbeck-Boussinesq approximation. This paper treats the onset of oscillations in a laterally infinite layer of fluid bounded by two horizontal planes. Two symmetrical sets of boundary conditions are used; free boundary conditions, which allow motion tangential to the bounding planes, and rigid boundary conditions, which do not. Attention is restricted to the oscillatory instability and small Prandtl numbers (ratio of kinematic viscosity to thermal diffusivity) relevant to dilute superfluid mixtures. A subsequent paper studies the effect of insulating sidewalls.

Our treatment of the onset of oscillations in a laterally unbounded slab extends previous theoretical work: For free boundary conditions, Busse ${ }^{6}$ used a long-wavelength expansion to infer a proportionality between frequency and amplitude and then calculated the onset amplitude numerically. Clever and Busse ${ }^{7}$ applied Galerkin's method to study both oscillating and stationary instabilities for rigid boundary conditions and small Prandtl numbers, obtaining good agreement with experiments.

Our formulation (Sec. II) relies on the vertical velocity, the vertical vorticity, and the temperature, which are convenient physical variables. It holds for any combination of rigid or free boundary conditions. A second-order expansion in the convective amplitude yields steady rolls, and superposed small perturbations then give the oscillations. In a Fourier expansion, the number of relevant normal modes is smaller than previously expected. ${ }^{8}$ The onset of oscillations occurs at nonzero wavelengths for rigid boundaries, in contrast to the long-wavelength (hydrodynamic) onset for free boundaries created by additional available modes. Since second-order terms in the amplitude expansion suffice to describe the basic physical properties, the oscillations are only mildly nonlinear.

Specializing to free boundary conditions (Sec. III), we provide a compact derivation of Busse's exact oscillation frequency. Our analytical calculation of the amplitude for onset of oscillations uses a six-mode truncation, which agrees with numerical work by Busse and ourselves to $2 \%$. Our explicit velocity and temperature fields predict streamline distortions during oscillations that may be observable.

A different approach is used in studying the more complicated rigid boundary conditions (Sec. IV), since we intend subsequently to consider bounded geometries, where the exact eigenfunctions are not known. Thus, we abandon the known eigenfunctions ${ }^{9}$ for an unbounded slab in favor of a truncated normal-mode expansion. This procedure requires only two terms for $1 \%-2 \%$ accuracy in the amplitude at onset of oscillations, compared with approximations including larger numbers of terms. Where the amplitude is small, our results compare well with the fully nonlinear Galerkin analysis by Clever and Busse.? Furthermore, the critical wavenumber for onset of oscillations $k_{c}$ turns out to be independent of the Prandtl number $\mathscr{P}$ for small $\mathscr{P}$. It is not known if this relation holds for laterally bounded geometries, where the sidewalls restrict $k_{c}$ to a denumerable set.

Good agreement is found with large-aspect-ratio experiments on air $(\mathscr{P}=0.71)$. Comparison with measurements on dilute superfluid ${ }^{3} \mathrm{He}-{ }^{4} \mathrm{He}$ mixtures in near unit aspect-ratio cells suggests that lateral boundaries or twofluid effects may be important (Sec. V).

\section{GENERAL FORMULATION}

In the Oberbeck-Boussinesq approximation, the classical one-component fluid equations are 


$$
\begin{aligned}
& \left(\nabla^{2}-\partial_{t}\right) \mathbf{v}+\hat{z} \theta-\nabla P=(\mathbf{v} \cdot \nabla) \mathbf{v}, \\
& \left(\nabla^{2}-\mathscr{P} \partial_{t}\right) \theta+\mathscr{R} \hat{z} \cdot \mathbf{v}=\mathscr{P} \mathbf{v} \cdot \nabla \theta, \\
& \boldsymbol{\nabla} \cdot \mathbf{v}=0 .
\end{aligned}
$$

These equations govern the deviations of velocity $\mathbf{v}$, temperature $\theta$, and pressure $P$ from the static conduction profile. Length, time, $v, \theta$, and $P$ are measured in units of $d, d^{2} / v, v /$ $d, v^{2} / g \alpha d^{3}$, and $\rho_{0} v^{2} / d^{2}$. Here $d, v, \rho_{0}, \alpha$, and $g$ are the depth, kinematic viscosity, mass density, coefficient of thermal expansion, and the gravitational acceleration. The Rayleigh number $\mathscr{R}=g \alpha d^{3} \Delta T / \kappa v$ characterizes the applied temperature gradient, where $\kappa$ is the thermal diffusivity, and $\Delta T$ is the (positive) temperature difference between lower and upper boundaries measured in conventional units. The resulting vertical density gradient drives the gravitational instability leading to convection. The only needed fluid property is the Prandtl number $\mathscr{P}=\nu / \kappa$.

We introduce two distinct conditions at the horizontal bounding planes which are readily expressed in terms of the vertical velocity $w=\hat{z} \cdot \mathbf{v}$ and vertical vorticity $\zeta$ $=(\nabla \times v) \cdot \hat{z}$. Common to both choices are the conditions

$$
w=\theta=0 \text {, }
$$

which confine the fluid between fixed isothermal planes. Free boundary conditions,

$$
\partial^{2} w=\partial \xi=0,
$$

allow motion tangential to the boundaries, whereas rigid boundary conditions,

$$
\partial w=\zeta=0,
$$

do not. Here, $\partial=\partial_{z}$ is the partial derivative in the vertical direction.

\section{A. Steady rolls}

We begin by studying the steady rolls, denoted by the uppercase letters $\mathbf{V}=(U, V, W)$. From Eqs. (1)-(3), the rolls evidently satisfy

$$
\begin{aligned}
& \nabla^{2} \mathbf{V}+\hat{z} \Theta-\nabla P=(\mathbf{V} \cdot \nabla) \mathbf{V}, \\
& \nabla^{2} \Theta+\mathscr{R} W=\mathscr{P} \mathbf{V} \cdot \boldsymbol{\nabla} \Theta, \\
& \boldsymbol{\nabla} \cdot \mathbf{V}=0 .
\end{aligned}
$$

The preferred mode of convection in layers with rigid boundaries ${ }^{10}$ is straight parallel rolls which break the translational symmetry of the static fluid layer. We take the direction of broken symmetry to be the $x$ axis and introduce a Fourier series in the $x$ coordinate:

$$
\mathbf{V}=\sum_{l=-\infty}^{+\infty} e^{i l q x} V_{l}(z), \quad \Theta=\sum_{l=-\infty}^{+\infty} e^{i l q x} \Theta_{l}(z)
$$

The nonzero components of $\mathbf{V}_{l}$ are related by continuity [see Eq. (9)]:

$$
U_{l}=(i / l q) \partial W_{l},
$$

which, coupled with the boundary condition (4), eliminates the horizontally uniform vertical velocity mode $\left(W_{0}=0\right)$. We choose the origin such that the column vector

$$
\Psi_{l}=\left(\begin{array}{c}
W_{l} \\
\Theta_{l}
\end{array}\right)=\Psi_{-l}
$$

is real and even in $l$, which ensures the reality of the velocity and temperature fields [Eq. (10)]. Equations (7) and (8) can be written wholly in terms of the vertical velocity and temperature modes:

$$
L_{l} \Psi_{l}=I_{l},
$$

where $\Psi_{l}$ is the eigenvector for mode $l$,

$$
L_{I}=\left(\begin{array}{cc}
\left(l^{2} q^{2}-\partial^{2}\right)^{2} l^{-2} q^{-2} & -1 \\
-1 & \left(l^{2} q^{2}-\partial^{2}\right) / \mathscr{R}
\end{array}\right)
$$

is a linear differential operator, and

$$
I_{l}=\sum_{l^{\prime}=-\infty}^{+\infty}\left(\begin{array}{c}
\left(l l^{\prime}\right)^{-1} q^{-2}\left(\partial^{3} W_{l}, W_{l-l^{\prime}}-\partial W_{l^{\prime}} \partial^{2} W_{l-l^{\prime}}\right)+\left(l^{\prime}\right)^{-1}\left(l-2 l^{\prime}\right) \partial W_{l^{\prime}}, W_{l-l^{\prime}} \\
\mathscr{P} \mathscr{R}^{-1}\left[\left(l^{\prime}\right)^{-1}\left(l-l^{\prime}\right) \partial W_{l^{\prime}} \Theta_{l-l^{\prime}}-W_{l}, \partial \Theta_{l-l^{\prime}}\right]
\end{array}\right)
$$

characterizes the nonlinearity. With the inner product $\left(\Psi_{l} \mid \Psi_{m}\right)$ defined as the vertical average of $\Psi_{l}^{T}(z) \Psi_{m}(z)$, the operator $L_{l}$ is self-adjoint,

$$
\left(\Psi_{k} \mid L_{l} \Psi_{m}\right)=\left(L_{l} \Psi_{k} \mid \Psi_{m}\right)
$$

for either set of boundary conditions.

To solve Eq. (13), we expand in powers of the roll amplitude $\epsilon$ :

$$
\begin{aligned}
& \Psi_{l}=\epsilon \Psi_{l}^{(1)}+\epsilon^{2} \Psi_{l}^{(2)}+\ldots, \\
& \mathscr{R}=\mathscr{R}^{(0)}+\epsilon \mathscr{R}^{(1)}+\epsilon^{2} \mathscr{R}^{(2)}+\ldots .
\end{aligned}
$$

To first (lowest) order,

$$
L_{l}^{(0)} \Psi_{l}^{(1)}=0 \text {, }
$$

where $L_{l}^{(0)}$ is formed from $L_{l}$ by replacing $\mathscr{R}$ by the eigenvalue $\mathscr{R}^{(0)}$. The basic roll pattern of wavenumber $q$ has $l= \pm 1$;

$$
L_{1}^{(0)} \Psi_{1}^{(1)}=0
$$

where $\Psi_{-1}^{(1)}=\Psi_{1}^{(1)}$ by Eq. (12), and $\psi_{l}^{(1)}=0$ for $l \neq \pm 1$. To second order,

$$
L_{l}^{(0)} \Psi_{l}^{(2)}=-L_{l}^{(1)} \Psi_{l}^{(1)}+I_{l}^{(2)}
$$

where

$$
L_{l}^{(1)}=\left(\begin{array}{lc}
0 & 0 \\
0 & -\mathscr{R}^{(1)}\left(l^{2} q^{2}-\partial^{2}\right) /\left(\mathscr{R}^{(0)}\right)^{2}
\end{array}\right) .
$$

Equation (15) shows that $I_{l}^{(2)}$ must vanish unless $l=0, \pm 2$. For $l=1$, the condition that the right-hand side of Eq. (20) be orthogonal to $\Psi_{1}^{(1)}$ then implies $\mathscr{R}^{(1)}=0$, leaving the simpler equation

$$
L_{l}^{(0)} \Psi_{l}^{(2)}=I_{l}^{(2)}
$$

for $l=0, \pm 2$. Thus, at second order, two modes are driven 
by the roll nonlinearity; the horizontally uniform $(l=0)$ mode,

$$
\partial \Theta_{0}^{(2)}=2 \mathscr{P}\left[W_{1}^{(1)} \Theta_{1}^{(1)}-\left(W_{1}^{(1)} \mid \Theta_{1}^{(1)}\right)\right],
$$

and the second horizontal harmonic $(l=2)$,

$$
L_{2}^{(0)} \Psi_{2}^{(2)}=I_{2}^{(2)},
$$

where

$$
I_{2}^{(2)}=\left(\begin{array}{c}
\frac{1}{2} q^{-2} \mathscr{W}\left[W_{1}^{(1)}, \partial^{2} W_{1}^{(1)}\right] \\
\mathscr{P} \mathscr{W}\left[\Theta_{1}^{(1)}, W_{1}^{(1)}\right] / \mathscr{R}^{(0)}
\end{array}\right)
$$

is the $l=2$ inhomogeneity and $\mathscr{W}$ is the Wronskian. By Eq. (17), the only nonzero modes through second order in $\epsilon$ are $\Psi_{ \pm 1}=\epsilon \Psi_{ \pm 1}^{(1)}, \Psi_{0}=\epsilon^{2} \Psi_{0}^{(2)}$, and $\Psi_{ \pm 2}=\epsilon_{ \pm 2}^{2} \Psi_{ \pm 2}^{(2)}$. The second-order Rayleigh number

$$
\begin{aligned}
\mathscr{R}^{(2)}\left(\Theta_{1}^{(1)} \mid W_{1}^{(1)}\right)= & \mathscr{R}^{(0)}\left(2 q^{2}\right)^{-1}\left(\boldsymbol{W}_{2}^{(2)} \mid \mathscr{W}\left[\boldsymbol{W}_{1}^{(1)}, \partial^{2} W_{1}^{(1)}\right]\right) \\
& +\mathscr{P}\left(\Theta_{2}^{(2)} \mid \mathscr{W}\left[\Theta_{1}^{(1)}, W_{1}^{(1)}\right]\right) \\
& +2 \mathscr{P}^{2}\left[\left(\Theta_{1}^{(1)^{2}} \mid \boldsymbol{W}_{1}^{(1)^{2}}\right)-\left(\Theta_{1}^{(1)} \mid \boldsymbol{W}_{1}^{(1)}\right)^{2}\right]
\end{aligned}
$$

follows from the orthogonality of the third-order inhomogeneity to the first-order solution. The resulting second-order amplitude expansion, valid for general boundary conditions on the functions $W(z)$ and $\Theta(z)$, is

$$
\begin{aligned}
\mathscr{R}=\mathscr{R}^{(0)} & +\epsilon^{2} \mathscr{R}^{(2)} \\
U(x, z)= & -2 \epsilon q^{-1} \sin q x \partial W_{1}^{(1)}(z) \\
& -\epsilon^{2} q^{-1} \sin 2 q x \partial W_{2}^{(2)}(z), \\
W(x, z)= & 2 \epsilon \cos q x W_{1}^{(1)}(z)+2 \epsilon^{2} \cos 2 q x W_{2}^{(2)}(z), \\
\Theta(x, z)= & 2 \epsilon \cos q x \Theta_{1}^{(1)}(z) \\
& +\epsilon^{2}\left[\Theta_{0}^{(2)}(z)+2 \cos 2 q x \Theta_{2}^{(2)}(z)\right] .
\end{aligned}
$$

This expansion should hold when the second term of Eq. (25a) is small compared to the first,

$$
\epsilon^{2} \mathscr{R}^{(2)} / \mathscr{R}^{(0)}=\left(\mathscr{R}-\mathscr{R}^{(0)}\right) / \mathscr{R}^{(0)}<1,
$$

which turns out to be satisfied at onset of oscillations (Secs. III and IV) for $q \leqslant q_{c}$ (the critical roll wavenumber at onset of convection) and $\mathscr{P}<1$. Since the roll amplitude takes the form

$$
\epsilon=\left(\frac{\mathscr{R}-\mathscr{R}^{(0)}(q)}{\mathscr{R}^{(2)}(\mathscr{P}, q)}\right)^{1 / 2}
$$

for $\mathscr{R}>\mathscr{R}^{(0)}$, and is zero below, the "order parameter" for onset of convection has critical exponent $1 / 2$. Because $\epsilon$ increases smoothly from zero, the bifurcation to convection is "normal," in contrast with divergent $\epsilon$ for an "inverted" bifurcation."

Equation (25) illustrates an important difference between rigid and free boundary conditions in the small Prandtl-number limit. For free boundary conditions, all second-order modes $\Psi_{l}^{(2)}$ will be seen (Sec. III) to vanish as $\mathscr{P} \rightarrow 0$, so that the onset of oscillations then coincides with the onset of convection. Thus, steady convection is impossible for a fluid layer of zero Prandtl number with free boundaries. For rigid boundaries, $\Psi_{2}^{(2)}$ remains nonzero as $\mathscr{P} \rightarrow 0$, leaving $\mathscr{R}^{(2)}$ finite in this limit. Hence, in contrast with free boundaries, rigid boundaries allow steady convection in fluids of zero Prandtl number.

\section{B. Perturbations}

To study the stability of steady rolls, we add small, time-dependent perturbations:

$$
\begin{aligned}
& \mathbf{v}(\mathbf{r}, t) \rightarrow \mathbf{V}(x, z)+\mathbf{v}^{\prime}(\mathbf{r}, t), \\
& \theta(\mathbf{r}, t) \rightarrow \Theta(x, z)+\theta^{\prime}(\mathbf{r}, t), \\
& P(\mathbf{r}, t) \rightarrow P(x, z)+P^{\prime}(\mathbf{r}, t) .
\end{aligned}
$$

Linearizing Eqs. (1)-(3) in the primed perturbations gives a homogeneous system with coefficients periodic in the $x$ direction:

$$
\begin{aligned}
& \left(\nabla^{2}-\partial_{t}\right) \mathbf{v}^{\prime}+\hat{z} \theta^{\prime}-\nabla P^{\prime}=\left(\mathbf{v}^{\prime} \cdot \nabla\right) \mathbf{V}(x, z)+[\mathbf{V}(x, z) \cdot \nabla] \mathbf{v}^{\prime} \\
& \left(\nabla^{2}-\mathscr{P} \partial_{t}\right) \theta^{\prime}+\mathscr{R} \hat{z} \cdot \nabla^{\prime}=\mathscr{P} \mathbf{v}^{\prime} \cdot \nabla \Theta(x, z)+\mathscr{P} \mathbf{V}(x, z) \cdot \nabla \theta^{\prime}, \\
& \nabla \cdot \mathbf{v}^{\prime}=0 .
\end{aligned}
$$

The primed variables must have exponential $y$ and $t$ dependences, and the $x$ dependence must be a plane wave multiplied by a periodic function (Floquet's theorem ${ }^{12}$ ). Consequently, expansions of the primed variables take the form

$\theta^{\prime}(\mathbf{r}, t)=\exp \left(i k_{x} x\right) \exp \left(i k_{y} y\right) \exp (\sigma t) \sum_{l=-\infty}^{+\infty} \exp (i l q x) \theta_{l}(z)$,

with similar expansions for $\mathbf{v}^{\prime}$ and $\zeta^{\prime}$. Continuity and the definition of vertical vorticity may be used to write Eqs. (29) and $(30)$ as

$$
\begin{aligned}
& \left(E_{l}+\sigma F_{l}\right) \psi_{l}=\sum_{l^{\prime}=-\infty}^{+\infty}\left(H_{l, l^{\prime}} \psi_{l-l^{\prime}}+I_{l, l^{\prime}} \xi_{l-l^{\prime}}\right), \\
& l=1,2, \ldots, \infty, \\
& \left(G_{l}+\sigma\right) \xi_{l}=\sum_{l^{\prime}=-\infty}^{+\infty}\left(J_{l, l}, \psi_{l-l^{\prime}}+K_{l, l^{\prime}} \xi_{l-l^{\prime}}\right), \\
& l=0,1, \ldots, \infty .
\end{aligned}
$$

Here

$$
\psi_{l}(z)=\left(\begin{array}{c}
w_{l}(z) \\
\theta_{l}(z)
\end{array}\right)
$$

and $\xi_{l}(z)$ are the desired perturbation modes,

$$
\begin{aligned}
E_{l} & =\left(\begin{array}{cc}
G_{l}^{2} / a_{l} & -1 \\
-1 & G_{l} / \mathscr{R}^{(0)}
\end{array}\right), \quad F_{l}=\left(\begin{array}{cc}
G_{l} / a_{l} & 0 \\
0 & \mathscr{P} / \mathscr{R}^{(0)}
\end{array}\right), \\
G_{l} & =a_{l}-\partial^{2},
\end{aligned}
$$

and $a_{l} \equiv\left(l q+k_{x}\right)^{2}+k_{y}^{2}$. The coupling elements on the right-hand side of Eq. (33) are known differential operators.

Experimentally, ${ }^{13}$ oscillations are periodic lateral disturbances that propagate along the roll axes ( $y$ direction). Accordingly, we henceforth take $k_{x}=0$ and $k \equiv k_{y}$. With this choice, the operators [Eqs. (35)] are even under $l \rightarrow-l$. The parity of the disturbances is found by allowing a periodic phase shift $x \rightarrow x+\delta x(y, t)$ in the steady rolls. For the temperature, for example,

$$
\Theta \rightarrow \sum_{l=-\infty}^{+\infty} e^{i l q x} \Theta_{l}(z)+i q \delta x(y, t)
$$




$$
\times \sum_{l=-\infty}^{+\infty} l e^{i l q x} \Theta_{l}(z)+\ldots,
$$

where the second term is the time-dependent temperature perturbation $\theta^{\prime}(\mathbf{r}, t)$ by Eq. (28b). Since the roll mode $\Theta_{l}(z)$ has even parity by choice [Eq. (12)], its corresponding perturbation mode $\theta_{l}(z)$ [(proportional to $\left.l \Theta_{l}(z)\right]$ must have odd parity. The other parities are found similarly, giving $\psi_{-l}(z)$ $=-\psi_{l}(z)$ and $\zeta_{-l}(z)=\zeta_{l}(z)$.

Equations (33) linearly couple an infinite number of perturbation modes. The couplings involve factors of order $\epsilon$ and higher due to the roll mode dependence of the coupling operators. To obtain a workable system, we retain only those horizontal couplings that involve terms of order $\epsilon^{2}$, which requires some care for free boundary conditions with vanishing $k$. This procedure isolates the most important perturbation modes at small roll amplitudes. Since the principal component of the steady rolls is $l=1$, we expect this component also to dominate the perturbations. To order $\epsilon^{2}$, the sums in Eqs. (33) are restricted to $l^{\prime}=0, \pm 1, \pm 2$. Accordingly, Eq. (33a) with $l=1$ gives

$$
\begin{aligned}
\left(E_{1}^{(0)}+\right. & \left.\sigma F_{1}^{(0)}\right) \psi_{1} \\
\approx & \epsilon^{2} H_{1,-2}^{(2)} \psi_{3}+\epsilon H_{1,-1}^{(1)} \psi_{2}+\epsilon^{2}\left(H_{1,0}^{(2)}-H_{1,2}^{(2)}\right) \psi_{1} \\
& +\epsilon_{1,-2}^{2} I_{1,-2}^{(2)} \zeta_{3}+\epsilon I_{1,-1}^{(1)} \zeta_{2}+\epsilon^{2}\left(I_{1,0}^{(2)}+I_{1,2}^{(2)}\right) \zeta_{1} \\
& +\epsilon I_{1,1}^{(1)} \zeta_{0},
\end{aligned}
$$

where the superscripts make explicit the order in $\epsilon$. The third term on the right-hand side of Eq. (37) is an important second-order self-coupling. The first order couplings to $\psi_{2}, \xi_{2}$, and $\xi_{0}$ lead us to consider the additional equations [from Eq. (33)],

$$
\begin{aligned}
& \left(E_{2}^{(0)}+\sigma F_{2}^{(0)}\right) \psi_{2} \approx \epsilon H_{2,1}^{(1)} \psi_{1}, \\
& \left(G_{2}^{(0)}+\sigma\right) \zeta_{2} \approx \epsilon J_{2,1}^{(1)} \psi_{1}, \\
& \left(G_{0}^{(0)}+\sigma\right) \zeta_{0} \approx 2 \epsilon J_{0,-1}^{(1)} \psi_{1}\left(+2 \epsilon K_{0,-1}^{(1)} \zeta_{1}\right),
\end{aligned}
$$

which produce couplings back to $\psi_{1}$. For rigid boundary conditions, $\psi_{3}, \zeta_{3}$, and $\xi_{1}$ occur only in higher-order contributions, and the corresponding form of Eq. (37) becomes

$$
\begin{aligned}
\left(E_{1}^{(0)}+\sigma F_{1}^{(0)}\right) \psi_{1} \approx & \epsilon\left(H_{1,-1}^{(1)} \psi_{2}+I_{1,-1}^{(1)} \zeta_{2}+I_{1,1}^{(1)} \zeta_{0}\right) \\
& +\epsilon^{2}\left(H_{1,0}^{(2)}-H_{1,2}^{(2)}\right) \psi_{1} .
\end{aligned}
$$

For rigid boundaries, the perturbation modes $\psi_{1}, \psi_{2}, \zeta_{0}$, and $\zeta_{2}$ will suffice to describe oscillations of the nonlinear (order $\epsilon^{2}$ ) rolls (Sec. IV). In contrast, for free boundaries (Sec. III), a consistent expansion to order $\epsilon^{2}$ requires the additional mode $\zeta_{1}$ in $\mathrm{Eq}$. (38c) because of the uniform vorticity satisfying Eq. (5).

\section{FREE BOUNDARY CONDITIONS}

Free boundary conditions at $z=0,1$ may be satisfied by a Fourier series in the $z$ coordinate. For the steady rolls, we take

$$
\Psi_{l}(z)=i \sum_{m=-\infty}^{+\infty} e^{i m \pi z} \Psi_{l, m},
$$

with $\Psi_{l_{s}-m}=-\Psi_{l, m}$ real. Note that $l$ and $m$ refer to $x$ and $z$, respectively. The Fourier series $(40)$ reduces the first-order problem (19) to a linear, homogeneous, algebraic system for each first-order roll amplitude $\Psi_{1, m}^{(1)}$. The lowest eigenvalue,

$$
\mathscr{R}^{(0)}(q)=\left(q^{2}+\pi^{2}\right)^{3} q^{-2},
$$

occurs for $m=1$. Minimizing $\mathscr{R}^{(0)}(q)$ gives the well-known critical roll wavenumber $q_{c}^{2}=\pi^{2} / 2$ and Rayleigh number $\mathscr{R}_{c}^{(0)}=27 \pi^{4} / 4$ for onset of convection. The eigenvector is

$$
\Psi_{l, m}^{(1)}=-\frac{m}{4 q^{2}}\left(\begin{array}{c}
q^{2} \\
\left(q^{2}+\pi^{2}\right)^{2}
\end{array}\right), \quad|l|=|m|=1,
$$

and is zero otherwise. For free boundary conditions, $\boldsymbol{W}_{1}^{(1)}$, $\partial^{2} W_{1}^{(1)}$, and $\Theta_{1}^{(1)}$ are all proportional (to $\sin \pi z$ ), so that the $l=2$ inhomogeneity $I_{2}^{(2)}$ in Eq. (23b) vanishes identically, Hence, by Eq. (22), only

$$
\Theta_{0, \pm 2}^{(2)}= \pm\left(\mathscr{P} / 16 \pi q^{2}\right)\left(q^{2}+\pi^{2}\right)^{2}
$$

enters to second order in the amplitude, and the secondorder Rayleigh number follows from Eq. (24),

$$
\mathscr{R}^{(2)}=\mathscr{P}^{2}\left[\left(q^{2}+\pi^{2}\right)^{2} / 8 q^{2}\right] .
$$

In summary, the steady two-dimensional rolls for free boundaries ${ }^{6}$ are given by

$$
\begin{gathered}
U(x, z)=-(\pi \epsilon / q) \sin q x \cos \pi z, \\
W(x, z)=\epsilon \cos q x \sin \pi z, \\
\Theta(x, z)=\frac{\epsilon\left(q^{2}+\pi^{2}\right)^{2}}{q^{2}} \cos q x \sin \pi z \\
\quad-\frac{\mathscr{P} \epsilon^{2}\left(q^{2}+\pi^{2}\right)^{2}}{8 \pi q^{2}} \sin 2 \pi z, \\
\mathscr{R}=\frac{\left(q^{2}+\pi^{2}\right)^{3}}{q^{2}}+\frac{\mathscr{P}^{2} \epsilon^{2}\left(q^{2}+\pi^{2}\right)^{2}}{8 q^{2}},
\end{gathered}
$$

with the quadratic terms proportional to powers of $\mathscr{P}$.

The boundary conditions on the perturbations $\psi_{l}(z)$ and $\zeta_{l}(z)$ may be satisfied by Fourier series analogous to Eq. (40) with expansion coefficients $\psi_{l, m}\left(\xi_{l, m}\right)$ odd (even) under $m \rightarrow-m$. Equation (33) yields a homogeneous algebraic system whose vanishing determinant of coefficients fixes the complex growth rate $\sigma$ as a function of $\epsilon, k, q$, and $\mathscr{P}$. At the critical roll amplitude for onset of oscillations $\epsilon_{0}(k, q, \mathscr{P})$, the real part of the growth rate $\operatorname{Re} \sigma$ passes from negative to positive, heralding the onset of oscillations. The corresponding $\operatorname{Im} \sigma$ gives the onset frequency $\omega_{0}(k, q, \mathscr{P})$.

The onset amplitude and frequency have been obtained numerically for various truncations. Case I, the simplest, retains the fundamental mode $\psi_{1,1}$ and the $l=0$ vorticities $\xi_{0,0}$ and $\zeta_{0,2}$, where cases II and III add the $\zeta_{1,1}(l=1)$ and $\zeta_{2,0}(l=2)$ vorticities, respectively. Cases II and III include all $\epsilon^{2}$ contributions; case III includes a single additional $\epsilon^{4}$ contribution $\left(\xi_{2,0}\right)$. Figure 1 shows the resulting onset amplitude at $q=q_{c}$ and $\mathscr{P}=0$. The critical amplitude $\epsilon_{c}(q, \mathscr{P})$ [the minimum of $\epsilon_{0}(k, q, \mathscr{P})$ with respect to $k$ ] occurs at $k=0$, hence oscillations are hydrodynamic for free boundary conditions. Evidently, $\epsilon^{2}$ contributions describe the hydrodynamic nature of oscillations; $\xi_{2,0}$ adds only quantitative information. To illustrate the accuracy of case III, we compare its critical amplitude $\epsilon_{c}\left(q_{c}, 0\right)=5.516$ with that of larger sets of modes $\psi_{l, m}, \zeta_{l, m}$ defined by $|l|,|m|<N$. Setting $N=2$ adds $\xi_{2,2}$ and $\psi_{2,2}$ to case III, and does not change the critical amplitude. The irrelevance of $\xi_{2,2}$ and $\psi_{2,2}$ as $k \rightarrow 0$ can also be shown analytically. For $N=3$ and $4, \epsilon_{c}\left(q_{c}, 0\right)$ 


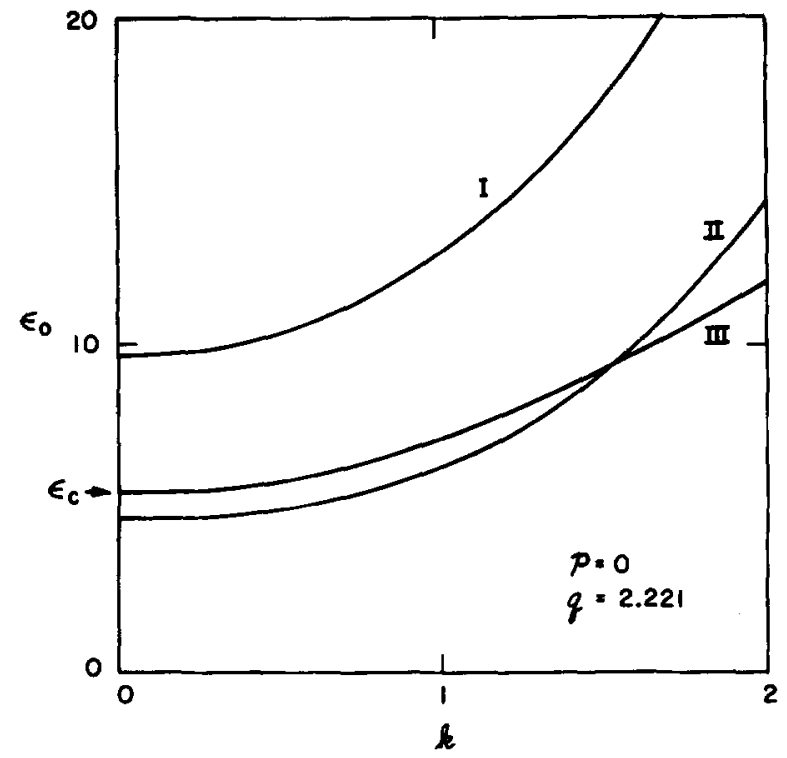

FIG. 1. Dependence of amplitude $\epsilon_{0}$ on wavenumber $k$ for onset of oscillations with free boundary conditions at the critical roll wavenumber $q_{c}=2.221$ and zero Prandtl number. Cases I, II, and III are successively better approximations.

$=5.421$ and 5.430, indicating rapid convergence for increasing values of $N$. Similar convergence is found for the onset amplitude $\epsilon_{0}(k, q, 0)$ over the range $0<k<2$. Since case III agrees with improved estimates to within $2 \%$ over $0<k<2$, we conclude that case III embraces the essential features of the oscillations. The critical amplitude $A_{i}$ $\equiv \epsilon_{c}\left(q_{c}, 0\right) / q_{c}^{2}=1.215$ given in Ref. 6 has recently been computed. ${ }^{14}$ The corrected value is $A_{i}=1.114$, or $\epsilon_{c}\left(q_{c}, 0\right)$ $=5.497$, in good agreement with our results.

It is instructive to study the case III modes $\psi_{1,1}, \zeta_{0,0}$, $\zeta_{0,2}, \zeta_{1,1}$, and $\zeta_{2,0}$ near critical conditions. Since the velocity must be finite and $\sigma$ must vanish as $k \rightarrow 0$, power series in the oscillation wavenumber $k$ take the forms $\psi_{l, m}=\psi_{l, m}^{[1]}$ $+\psi_{l, m}^{[2]}+\ldots, \zeta_{l, m}=\zeta_{l, m}^{[0]}+\zeta_{l, m}^{[1]}+\ldots$, and $\sigma=\sigma^{[1]}+\sigma^{[2]}$ $+\ldots$. A perturbation expansion (similar to the amplitude expansion of Sec. II) yields the Busse oscillation frequency

$$
\sigma^{[1]}= \pm i(\pi / 2)(k / q) \epsilon \equiv \pm i \omega
$$

in first order, and the growth rate

$$
\begin{aligned}
2(1+ & \mathscr{P}) q^{2} \frac{\sigma^{[2]}}{k^{2}} \\
= & -\left[(3+\mathscr{P}) q^{2}-\pi^{2}\right]+\frac{\epsilon^{2}\left[(3-\mathscr{P}) \pi^{2}-(1+\mathscr{P}) q^{2}\right]}{32\left(q^{2}+\pi^{2}\right)} \\
& +\frac{\pi^{2} \epsilon^{2}(1+\mathscr{P})}{4\left(q^{2}+\pi^{2}+\pi^{2} \epsilon^{2} / 8 q^{2}\right)}
\end{aligned}
$$

in second order. Busse obtains a result similar to (47) using an expansion to order $\epsilon^{4}$, whereas we retain $\epsilon^{2}$ explicitly in the denominator. Since this contribution (from $\xi_{2,0}$ ) is of relative order 0.5 , his expansion converged slowly and was replaced by a numerical computation involving many normal modes. The offending vertically uniform $\xi_{2,0}$ mode is absent for rigid boundary conditions, so that an amplitude expansion is more relevant in that case. Equation (47) is rich in information. For small $\epsilon$, the first term on the right-hand

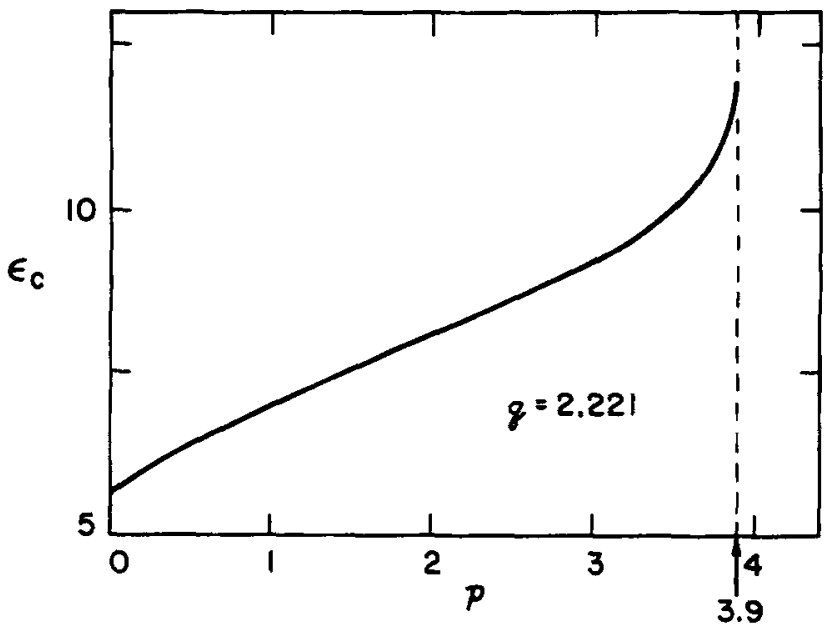

FIG. 2. Prandtl number dependence of the critical amplitude for onset of oscillations $\epsilon_{c}$ at the critical roll wave vector $q_{c}=2.221$ for free boundary conditions.

side dominates, and $\sigma^{[2]}$ is negative, indicating stability of convective rolls. Setting $\sigma^{[2]}=0$ yields a quadratic in the square of the critical amplitude $\epsilon_{c}(q, \mathscr{P})$, which readily produces an explicit, analytical expression for $\epsilon_{c}(q, \mathscr{P})$. As expected, the value $\epsilon_{c}\left(q_{c}, 0\right)=5.516$ confirms the case III value obtained numerically. Furthermore, the relation describes the dependence on Prandtl number (Fig. 2): $\epsilon$ increases with $\mathscr{P}$ until $\mathscr{P}_{c}=3.9$, where the slope of the critical amplitude becomes infinite, while the function itself remains finite. Above $\mathscr{P}_{c}$, the critical amplitude $\epsilon_{c}^{2}\left(q_{c}, \mathscr{P}\right)$ is imaginary. Hence, $\mathscr{P}_{c}$ is an upper Prandtl number limit for the onset of oscillations with free boundary conditions in this approximate description. Since $\epsilon_{\mathrm{c}}^{2} \mathscr{R}^{(2)} / \mathscr{R}^{(0)} \propto \mathscr{P}^{2}$ for free boundary conditions, our small amplitude results for the critical Rayleigh number for onset of oscillations $\mathscr{R}_{c}=\mathscr{R}^{(0)}+\epsilon_{c}^{2} \mathscr{R}^{(2)}$ are exact as $\mathscr{P} \rightarrow 0$. At $\mathscr{P} \sim 1$ and $q=q_{c}, \epsilon_{c}^{2} \mathscr{R}^{(2)} / \mathscr{R}^{(0)} \sim 1$ can no longer be considered small, so that $\mathscr{P} \gtrsim 1$ in Fig. 2 is outside the strict region of validity of the theory.

By the considerations of Sec. II, the $l=1$ vorticity $\zeta_{1,1}$ should not have been important. In fact, for free boundary conditions it enters in order $\epsilon^{2}$ because of the allowed vertically constant $\xi_{0,0}$ mode. Acting on this mode and for small $k$, the operator $G_{0}^{(0)}+\sigma$ is proportional to $\epsilon$ [see Eq. (46)] which couples $\zeta_{0}(z)$ to $\zeta_{1}(z)$ in zeroth order by Eq. (38c). In turn, $\zeta_{1}(z)$ couples to $\xi_{0}(z)$ in first order which gives an overall contribution of order $\epsilon^{2}$ to the determinant at long wavelengths. Rigid boundary conditions eliminate the $\zeta_{0,0}$ mode, so $\zeta_{1,1}$ may be neglected regardless of the value of $k$.

We can now construct the total velocity and temperature fields to order $k^{2}$ using the case III modes. Guided by experiments, ${ }^{13}$ we construct running rather than standing waves at onset $(\operatorname{Re} \sigma=0)$ by adding the real part of Eqs. (32) with $\sigma=-i \omega_{0}$ and $\epsilon=\epsilon_{0}$ to the steady roll pattern [Eqs. (45)]:

$$
\begin{aligned}
u(\mathbf{r}, t)= & -\pi q^{-1} \epsilon_{0} \sin \{q[x+\delta x(y, t)]\} \cos \pi z \\
& -\partial_{t} \delta x(y, t)+k^{2}\left(\frac{q^{2}+\pi^{2}-\pi^{2} \epsilon_{0}^{2} / 8 q^{2}}{q^{2}+\pi^{2}+\pi^{2} \epsilon_{0}^{2} / 8 q^{2}}\right.
\end{aligned}
$$




$$
\begin{aligned}
& \left.-\frac{\epsilon_{0}^{2}}{16 q^{2}} \cos 2 \pi z\right) \delta x(y, t), \\
v(\mathbf{r}, t)= & 2 \sin q x \cos \pi z \partial_{t} \delta x(y, t) \\
& +\frac{\pi^{2} k^{2} \epsilon_{0}^{2}\left[\sin q x \cos \pi z+\left(\pi \epsilon_{0} / 8 q^{2}\right) \sin 2 q x\right]}{2 q^{2}\left(q^{2}+\pi^{2}+\pi^{2} \epsilon_{0}^{2} / 8 q^{2}\right)} \\
& \times \delta x(y, t), \\
w(\mathbf{r}, t)= & \epsilon_{0} \cos \left\{q\left[x+\left(1+k^{2} / q^{2}\right) \delta x(y, t)\right]\right\} \sin \pi z, \\
\theta(\mathbf{r}, t)= & \left(q^{2}+\pi^{2}\right)^{2} q^{-2}\left[w(\mathbf{r}, t)-\mathscr{P} \epsilon_{0}^{2}(8 \pi)^{-1} \sin 2 \pi z\right],
\end{aligned}
$$

where

$$
\delta x(y, t)=\delta x_{m} \sin \left(k y-\omega_{0} t\right) .
$$

Equations $(48)$ give the deviations of velocity $\mathbf{v}=(u, v, w)$ and the temperature $\theta$ from the static conduction state describing infinitesimal amplitude $\left(\delta x_{m}<1\right)$ oscillations to order $k^{2}$ using the case III truncation. It is remarkable that this fourdimensional $(\mathbf{r}, t)$ flow can be constructed analytically. Based on the accuracy of the critical amplitude $\epsilon_{0}$, Eqs. (48) are expected to hold to approximately $2 \%$. Setting the oscillation amplitude $\delta x_{m}$ to zero recovers straight parallel rolls [Eq. (45)], as expected. Figure 3(a) shows the form of the roll

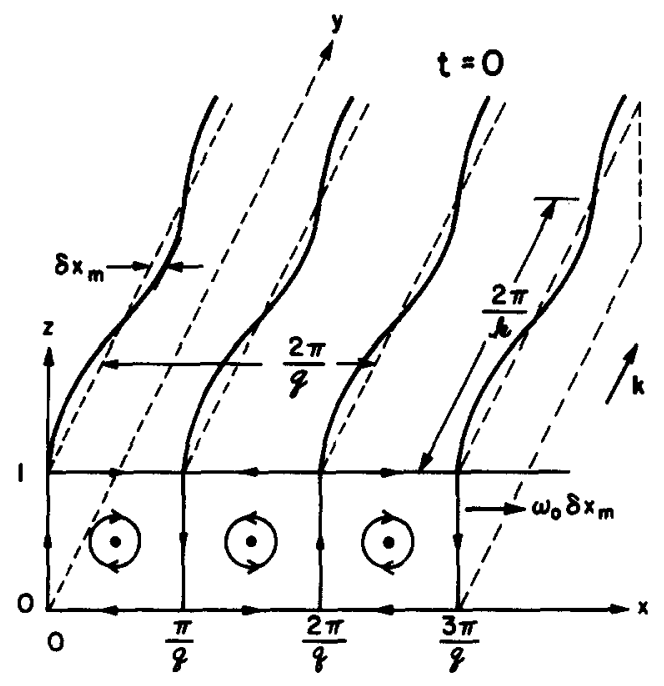

(a)

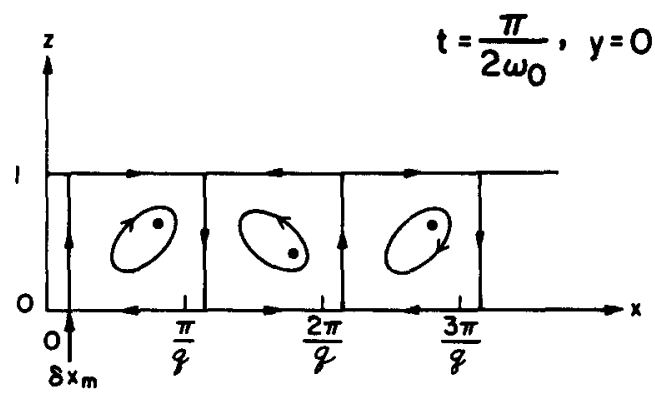

(b)

FIG. 3. Oscillation pattern for free boundary conditions at $t=0$ (a) and $t=\pi / 2 \omega_{0}$ (b). Transverse oscillations propagate along $\mathbf{k}=k \hat{y}$ (roll axis) with amplitude $\delta x_{m}$, wavelength $2 \pi / k$, and frequency $\omega_{0}$, where the oscillation wavelength has been compressed for clarity. The arrows indicate the circulation pattern in the $y=0$ cross section, which has uniform transverse velocity $\omega_{0} \delta x_{m}$ at $t=0$ and is approximately stationary at $t=\pi / 2 \omega_{0}$. Roll boundaries at $t=\pi / 2 \omega_{0}$ are ill-defined due to small $\left(\propto k^{2}\right)$ distortions, which are not shown. distortions at $t=0$. Owing to the second term of Eq. (48a), the $y=0$ cross section of fluid has instantaneous uniform velocity $\omega_{0} \delta x_{m}$ in the $+x$ direction. The remaining terms of Eqs. (48a)-(48c) give the basic circulation pattern (indicated by arrows) with nodes (1) at the center of the rolls. Roll boundaries are indicated by vertical segments at $y=0$. In a time $t=\pi / 2 \omega_{0}$, the wave propagates a distance $\pi / 2 k$ in the $+y$ direction, and the $y=0$ cross section is at maximum displacement [Fig. 3(b)], for which the $k^{2}$ terms of Eqs. (48a)(48c) shift the nodes in the $(+x, \pm z)$ directions and make roll boundaries ill-defined because of nonuniform $(\propto \sin 2 \pi z)$ lateral motions. Hence, linear stability analysis of oscillations predicts that each roll cross section (at fixed $y$ ) oscillates roughly harmonically in the $x$ direction. The temperature [Eq. (48d)] is enhanced at updrafts (roll boundaries with $w>0$ ), as expected. For rigid boundary conditions, the motion at $z=0,1$ is disallowed, but is otherwise similar.

\section{RIGID BOUNDARY CONDITIONS}

To satisfy rigid boundary conditions for an infinite slab, we expand the horizontal roll modes $\Psi_{1}^{(1)}(z)$ and $\Psi_{2}^{(2)}(z)$ of Sec. II in a complete set of basis functions. Although previous calculations ${ }^{9,10}$ have used the exact roll modes, our procedure also applies to laterally bounded geometries where the exact eigenfunctions are not known.

To solve the first-order problem [Eq. (19)], we write components of $\Psi_{1}^{(1)}$ as a series:

$$
\begin{aligned}
& W_{1}^{(1)}(z)=\sum_{m=1}^{\infty} W_{1, m}^{(1)} C_{m}(z), \\
& \Theta_{1}^{(1)}(z)=\sum_{m=1}^{\infty} \Theta_{1, m}^{(1)} \chi_{m}(z),
\end{aligned}
$$

where $C_{m}(z)$ and $\chi_{m}(z)$ are even orthonormal solutions of

$$
\begin{aligned}
& \partial^{4} C_{m}(z)=\lambda_{m}^{4} C_{m}(z), \\
& \partial^{2} \chi_{m}(z)=-\alpha_{m}^{2} \chi_{m}(z),
\end{aligned}
$$

subject to the rigid boundary conditions $C_{m}=\partial C_{m}$ $=\chi_{m}=0$ at $z= \pm 1 / 2$. Here, $W_{1,1}^{(1)}=1$ defines our normalization, $\chi_{m}(z)=\sqrt{2} \cos (2 m-1) \pi z$, and the eigenvalues $\lambda_{m}$ are tabulated by Chandrasekhar. ${ }^{9}$ Substitution into Eq. (19) readily yields a homogeneous algebraic system with matrix elements that may be calculated analytically:

$$
\begin{gathered}
\sum_{m=1}^{\infty}\left\{\left[\left(q^{4}+\lambda_{m}^{4}\right) q^{-2} \delta_{l m}+2\left(C_{l}^{\prime} \mid C_{m}^{\prime}\right)\right] W_{1, m}^{(1)}\right. \\
\left.-\left(C_{l} \mid \chi_{m}\right) \Theta_{1, m}^{(1)}\right\}=0, \quad l=1,2, \ldots, \infty,
\end{gathered}
$$

$\sum_{m=1}^{\infty}\left\{-\left(\chi_{l} \mid C_{m}\right) W_{1, m}^{(1)}+\left(q^{2}+\alpha_{m}^{2}\right) \mathscr{R}^{(0)^{-1}} \delta_{l m} \Theta_{1, m}^{(1)}\right\}=0$,

$l=1,2, \ldots, \infty$.

Here $C_{i} \equiv \partial C_{l}$, and $(A \mid B)$ is the vertical average of the product $A B$, as before. Approximating the roll modes by one term in the series (49) predicts the critical Rayleigh number $\mathscr{R}_{c}^{(0)}=1728$ and wavenumber $q_{c}=3.097$. A two-term approximation yields the improved values $\mathscr{R}_{c}^{(0)}=1709$ and $q_{c}$ 
$=3.114$, which agree well with the values $\mathscr{R}_{c}^{(0)}=1708$ and $q_{c}=3.117$ obtained using the exact eigenfunctions. ${ }^{9}$

Since the second-order inhomogeneity [Eq. $(23 \mathrm{~b})]$ is odd at about $z=0$, we write the components of $\Psi_{2}^{(2)}$ as

$$
\begin{aligned}
& W_{2}^{(2)}=\sum_{m=1}^{\infty} W_{2, m}^{(2)} S_{m}(z), \\
& \Theta_{2}^{(2)}=\sum_{m=1}^{\infty} \Theta_{2, m}^{(2)} \phi_{m}(z),
\end{aligned}
$$

where $S_{m}$ and $\phi_{m}$ are the odd counterparts of $C_{m}$ and $\chi_{m}$, respectively. Substitution into Eq. (23a) yields an inhomogeneous algebraic system in the coefficients $W_{2, m}^{(2)}$ and $\Theta_{2, m}^{(2)}$, and the second-order Rayleigh number $\mathscr{R}^{(2)}(q, \mathscr{P})$ follows from Eq. (24) by numerical integration. For reference, our one-, two-, and three-term approximations give $\mathscr{R}^{(2)}\left(q_{c}\right.$, $0.025)=1.900,1.438$, and 1.467 , which compare well with the corresponding value 1.479 using the exact eigenfunctions. ${ }^{10}$

Onset of oscillations can now be studied to order $\epsilon^{2}$ by using the horizontal modes $\psi_{1}, \psi_{2}, \zeta_{0}$, and $\zeta_{2}$ (Sec. II). Substituting appropriate expansions into the coupled system of Eqs. (38) and (39), multiplying by appropriate basis functions and integrating yields a homogeneous algebraic system in the unknown expansion coefficients. The resulting onset amplitude at the critical roll wavenumber $q_{c}=3.114$ is plotted in Fig. 4 for $\mathscr{P}=0.12$, which pertains to recent experiments ${ }^{4}$ in dilute superfluid ${ }^{3} \mathrm{He}-{ }^{4} \mathrm{He}$ mixtures. In contrast with free boundary conditions (Fig. 1), the onset amplitude $\epsilon_{c}$ attains a minimum at finite oscillation wavenumber $k_{c}$. Retaining three terms in the roll and perturbation expansions alters $\epsilon_{\mathrm{r}}$ from its two-term approximation by only $1 \%$, so that the two-term approximation is adequate for most purposes. For a specified roll amplitude $\epsilon>\epsilon_{c}$, possible oscillation wavenumbers lie in a band defined by the neutral stability curve $\epsilon_{0}$. (See example for $\epsilon=15$, Fig. 4.) For $\epsilon \gtrsim 16$

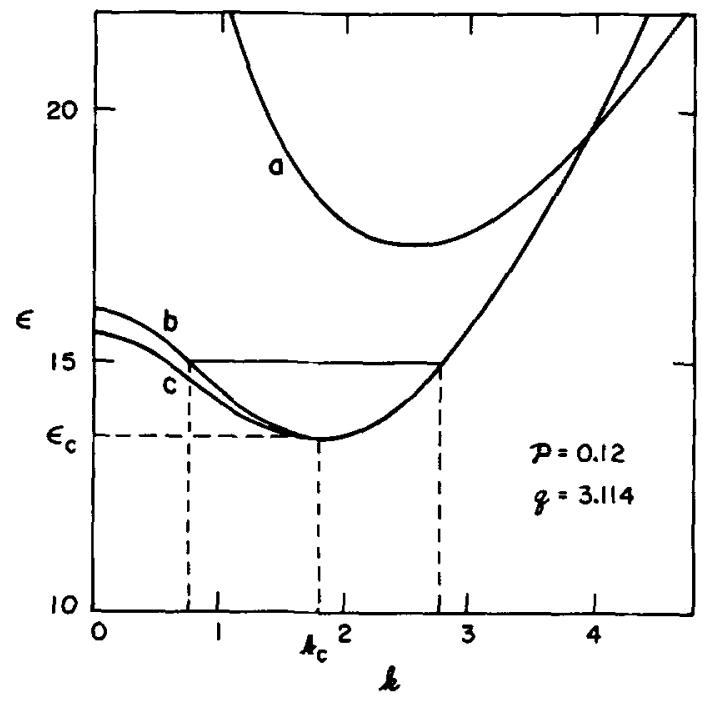

FIG. 4. Dependence of amplitude $\epsilon_{0}$ on wavenumber $k$ for onset of oscillations with rigid boundary conditions at the critical roll wavenumber $q_{c}=3.114$ and Prandtl number $\mathscr{P}=0.12$. The critical amplitude $\epsilon_{c}$ is the minimum of $\epsilon_{0}$. Curves $a, b$, and c denote one-, two-, and three-term expansions.

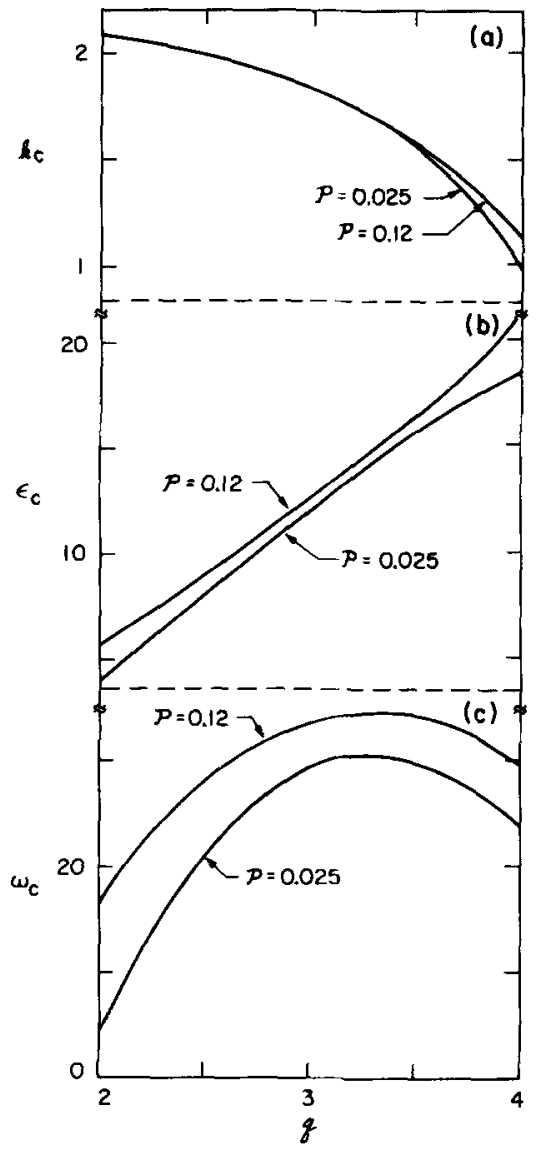

FIG. 5. Dependence of critical oscillation wavenumber $k_{c}(\mathrm{a})$, amplitude $\epsilon_{c}$ (b), and frequency $\omega_{\mathfrak{c}}$ (c) on roll wavenumber $q$ for rigid boundaries and Prandtl numbers $\mathscr{P}=0.025$ and 0.12 .

$(\mathscr{R} \gtrsim 2500)$, the allowed band extends to $k=0$; hence linear stability allows long wavelength oscillations for rigid boundary conditions at small Prandtl number. In the two-term approximation, Fig. 5 shows the dependences of the critical wavenumber $k_{c}$, amplitude $\epsilon_{c}$, and frequency $\omega_{c}$ for $\mathscr{P}=0.12$ and 0.025 on the roll wavenumber. (Here $\mathscr{P}=0.025$ pertains to mercury.) The onset amplitudes and critical wavenumbers are new. Clever and Busse ${ }^{7}$ calculate frequencies for choices of the oscillation wavenumber, but no critical wavenumbers $k_{c}$ are found in the literature. ${ }^{7,15}$ The onset amplitude cannot be deduced from Clever and Busse without knowledge of $\mathscr{R}^{(2)}$. The critical amplitude vanishes at some value of $q<2$; only $q$ values relevant to oscillations at $\mathscr{P}=0.025,0.12$ are retained in Fig. $5(\mathrm{~b})$. The critical frequency measured in Hertz is given by $v \omega_{c}(q, \mathscr{P}) /$ $2 \pi d^{2}$.

The critical Rayleigh number for onset of oscillations $\mathscr{R}_{c}=\mathscr{R}^{(0)}+\epsilon_{c}^{2} \mathscr{R}^{(2)}$ and the Rayleigh number for onset of convection $\mathscr{R}^{(0)}$ are plotted in Fig. 6. For $\mathscr{R}>\mathscr{R}_{c}$, infinitesimal amplitude oscillations grow with time, so for fixed $\mathscr{R}$, oscillations are restricted to a band of roll wavenumbers bounded by the intersections of $\mathscr{R}_{c}(\mathscr{P}, q)$ with $\mathscr{R}$. The shift in the minimum of $\mathscr{R}_{c}$ indicates that smaller $q$ values are relevant as $\mathscr{P}$ increases. The dashed trace for $\mathscr{P}=0.025$ of Clever and Busse 7 is included in Fig. 6 for comparison. They obtain full Galerkin solutions to the problem, where we have worked only to order $\epsilon^{2}$. Evidently, the two methods agree 


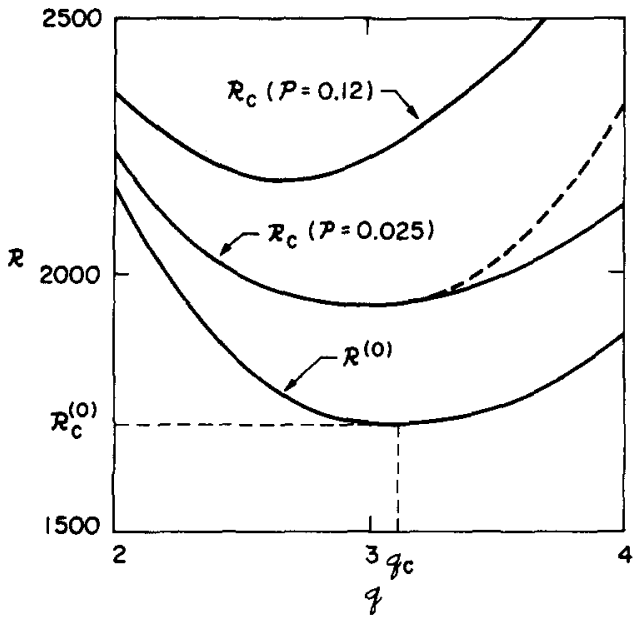

FIG. 6. Dependence of Rayleigh number for onset of oscillations $\mathscr{R}_{c}$ and convection $\mathscr{R}^{(0)}$ on roll wavenumber $q$ for rigid boundary conditions and Prandtl numbers $\mathscr{P}=0.025$ and 0.12 . For $\mathscr{R}<\mathscr{R}^{(0)}$, the static conduction state is stable. Below $\mathscr{R}_{c}$, steady rolls are stable against oscillations. $\mathscr{R}_{c}^{(0)}$ is the minimum of $\mathscr{R}^{(0)}$. The dashed trace for $\mathscr{P}=0.025$ was obtained by Clever and Busse (Ref. 7).

very well on the location and value of the minimum, and differ only for $q \gtrsim 3.2$, where $\epsilon_{c}^{2} \mathscr{R}^{(2)} / \mathscr{R}^{(0)} \approx 0.14$ (ratio of terms in $\mathscr{R}_{c}$, assumed small) becomes appreciable [see Fig. $5\left(\right.$ b)]. As for free boundaries, $\epsilon_{c}^{2} \mathscr{R}^{(2)} / \mathscr{R}^{(0)}$ rises to order unity as $\mathscr{P}$ approaches unity (at $q=q_{c}$ ), so that the small $\epsilon$ expansion is limited to $\mathscr{P} \leqslant 1$. The effect of increased $\epsilon$ on the frequency is more pronounced; at $\mathscr{P}=0.12$, for example, the ratio of their critical frequency $\sigma_{i} / \mathscr{P}$ to ours rises from approximately 1.6 at $q=2.2$ to approximately 2.2 at $q=3.114$, indicating agreement between the two methods for small $q$ (small $\epsilon$ ). Clever and Busse report frequencies for $q \geqslant 2.2$ only, but an extrapolation to $q=1.9$ at $\mathscr{P}=0.71$ (pertinent for air, see Sec. V) gives approximately unity for the frequency ratio.

An unexpected result of our calculation (see Fig. 5) is the approximate independence of the critical oscillation wavenumber on the Prandtl number for small Prandtl numbers $(\mathscr{P} \leqslant 0.12)$; it holds while the small $\epsilon$ assumption is $\operatorname{good}(q \leqslant 3.5)$. Accordingly, the location of the minimum of the onset amplitude $\epsilon_{0}(k, q, \mathscr{P})$ with respect to $k$ (Fig. 4) depends only on the roll wavenumber, whereas the value at the minimum depends on $\mathscr{P}$. This property is shared trivially with free boundary conditions in a laterally unbounded slab, where $k_{c} \equiv 0$. The prediction is directly verifiable experimentally; oscillations first appear at the critical wavenumber. It remains to see if $k_{c}$ is independent of $\mathscr{P}$ for bounded geometries, where $k_{c}$ is restricted to a discrete set by the shape of the sidewalls.

The small-amplitude procedure used above describes previous results and leads to new results as well, and hence can be applied with guarded confidence to bounded geometries.

\section{DISCUSSION}

Willis and Deardorf $\mathrm{f}^{13}$ observed oscillations in a square cell of aspect ratio $\Gamma=15.7$ (ratio of half the largest horizon- tal dimension to the depth) filled with air $(\mathscr{P}=0.71)$ and a small amount of smoke for visualization. Using a typical roll wavenumber $q=1.9$ from their top-view photograph, Fig. $3(\mathrm{a})$, we find the critical conditions $\mathscr{R}_{c}=5900, \omega_{c}=29$, and $k_{c}=2.38$. (Computations with other values of $q$ at $\mathscr{P}=0.71$ were not made.) Although the perturbation expansion is not strictly valid $\left(\epsilon_{c}^{2} \mathscr{R}^{(2)} / \mathscr{R}^{(0)} \sim 1.5\right)$, these compare favorably with the measurements $\mathscr{R}_{c}=5800$ and $\omega_{c}=27$. The oscillation wavenumber $k$ was measured only for fully developed rolls. At $\mathscr{R}=9000$, the value $k=2.2$ [their Fig. 3(a)] easily falls within the allowed band of oscillation wavenumbers $1.2 \leqslant k \leqslant 4.0$, and is even close to our critical value $k_{c}=2.38$. Clearly, theory and experiment agree well for this large-aspect-ratio classical system.

Experiments by Maeno, Haucke, and Wheatley in dilute superfluid ${ }^{3} \mathrm{He}-{ }^{4} \mathrm{He}$ mixtures near $T=1 \mathrm{~K}$ study the frequency and Rayleigh number at onset of oscillations in a cylindrical cell ${ }^{4}$ of aspect ratio (radius to depth ratio) $\Gamma=1.2$ and a rectangular cell ${ }^{5}$ of aspect ratio $\Gamma=1.0$. They measure an increase in Rayleigh number with Prandtl number, consistent with Fig. 6. Other similarities with a classical one-component fluid are discussed in Ref. 5. However, for either geometry, measured critical Rayleigh numbers $\mathscr{R}_{c}$ $\approx 10000$ and frequencies $\omega_{c} \approx 250$ for onset of oscillations exceed typical values $\mathscr{R}_{c} \approx 2000$ and $\omega_{c} \approx 30$ for a laterally unbounded classical fluid layer. These differences motivate the inclusion of sidewalls and two-fluid effects in the theory, for which an amplitude expansion may be insufficient. This problem is currently under investigation.

\section{ACKNOWLEDGMENT}

This work was supported in part by National Science Foundation Grant No. DMR 81-18386.

'P. A. Warkentin, H. J. Haucke, and J. C. Wheatley, Phys. Rev. Lett. 45, 918 (1980); P. A. Warkentin, H. J. Haucke, P. Lucas, and J. C. Wheatley, Proc. Nat1. Acad. Sci. U.S.A. 77, 6983 (1980).

${ }^{2}$ V. Steinberg, Phys. Rev. A 24, 975 (1981); V. Steinberg and H. R. Brand, Phys. Rev. B 28, 1618 (1983).

${ }^{3}$ A. L. Fetter, Phys. Rev. B 26, 1164 and 1174 (1982).

${ }^{4} \mathrm{Y}$. Maeno, H. Haucke, and J. Wheatley, in AIP Conference Proceedings, edited by E. D. Adams and G. G. Ihas (American Institute of Physics, New York, 1983), p. 412.

${ }^{5}$ Y. Maeno, H. Haucke, and J. Wheatley (private communication).

${ }^{6} \mathrm{~F}$. H. Busse, J. Fluid. Mech. 52, 97 (1972).

${ }^{7}$ R. M. Clever and F. H. Busse, J. Fluid. Mech. 65, 625 (1974).

${ }^{8}$ G. Ahlers and R. P. Behringer, Prog. Theor. Phys. Suppl. 64, 186 (1978).

${ }^{9} \mathrm{~S}$. Chandrasekhar, Hydrodynamic and Hydromagnetic Stability (Clarendon, Oxford, 1961), Chap. II.

${ }^{10}$ A. Schlüter, D. Lortz, and F. Busse, J. Fluid. Mech. 23, 129 (1965).

"I. B. McLaughlin and P. C. Martin, Phys. Rev. A 12, 186 (1975).

${ }^{12}$ E. T. Whittaker and G. N. Watson, $A$ Course in Modern Analysis (Cambridge U. P., London, 1962), Chap. XIX.

${ }^{13}$ G. E. Willis and J. W. Deardorff, J. Fluid. Mech. 44, 661 (1970).

${ }^{14} \mathrm{E}$. W. Bolton and F. H. Busse (private communication).

${ }^{15}$ R. M. Clever and F. H. Busse, Z. Angew. Math. Phys. [J. Appl. Math. Phys.] 29, 711 (1978); F. H. Busse and R. M. Clever, J. Fluid. Mech. 91, 319 (1979); F. H. Busse, Rep. Prog. Phys. 41, 1929 (1978). 